\title{
Compare and Correlate the Presence of Bacterial Pathogen Concentrations and Glycaemic Status in Type 2 Diabetic and Non-Diabetic Patients with Chronic Periodontitis Following Phase 1 Therapy
}

\author{
Amit Lakhani ${ }^{1}$, Ena Sharma², Surinder Sachdeva ${ }^{3}$, Supreet Thind ${ }^{4}$, Karun Chaudhary ${ }^{5}$, Savita Kapila ${ }^{6}$
}

\begin{abstract}
${ }^{1}$ Department of Orthopaedics, Maharishi Markandeshwar Medical College and Hospital Kumarhatti, Solan, Himachal Pradesh, India. ${ }^{2}$ Department of Periodontics, Maharishi Markandeshwar College of Dental Sciences and Research, Mullana, Haryana, India. ${ }^{3}$ Department of Periodontics, Maharishi Markandeshwar College of Dental Sciences and Research, Mullana, Haryana, India. ${ }^{4}$ Department of Periodontics, Maharishi Markandeshwar College of Dental Sciences and Research, Mullana, Haryana, India. ${ }^{5}$ Department of Periodontics, Maharishi Markandeshwar College of Dental Sciences and Research, Mullana, Haryana, India. ${ }^{6}$ Department of Medicine, Maharishi Markandeshwar Institute of Medical Sciences and Research, Mullana, Haryana, India.
\end{abstract}

\section{ABSTRACT}

\section{BACKGROUND}

At present, a bidirectional pathway has been suggested between DM and periodontitis, since patients with DM are more predisposed to periodontal diseases, the established periodontitis may simultaneously impair adequate glycaemic control. We wanted to study the change in bacterial pathogen concentrations in plaque as confirmed polymerize chain reaction (PCR) in patients with and without type- 2 diabetes before and after phase- 1 therapy.

\section{METHODS}

Subgingival plaque samples were collected from the deepest part of the pocket by using a sterile curette in 2 different Eppendorf tubes containing tris-EDTA buffer. The plaque samples were then subjected to PCR for detecting bacteria (P. gingivalis, T. forsythia, P. intermedia).

\section{RESULTS}

In-vitro microbiological assessment (PCR analysis) showed significant reduction of bacteria within both diabetic and non-diabetic groups. In contrast, the intergroup comparison revealed no significant differences in the microbial counts in both the diabetic and non-diabetic groups thus suggesting that phase-1 therapy may not alter the pathogenic microflora in chronic periodontitis patients with or without diabetes.

\section{CONCLUSIONS}

In-vitro microbiological assessment (PCR analysis) showed significant reduction of bacteria within both diabetic and non-diabetic groups. In contrast, the intergroup comparison revealed no significant differences in the microbial counts in both the diabetic and non-diabetic groups thus suggesting that phase-1 therapy may not alter the pathogenic microflora in chronic periodontitis patients with or without diabetes.

\section{KEY WORDS}

Chronic Periodontitis, PCR, Oral Pathogens, Diabetes
Corresponding Author:

Dr. Ena Sharma,

Department of Periodontics,

Maharishi Markandeshwar College of

Dental Sciences and Research,

Mullana, Haryana, India.

E-mail:dr.sharma_ena@yahoo.co.in

DOI: $10.14260 /$ jemds/2019/860

Financial or Other Competing Interests: None.

How to Cite This Article:

Lakhani A, Sharma E, Sachdeva S, et al. Compare and correlate the presence of bacterial pathogen concentrations and glycaemic status in type 2 diabetic and non-diabetic patients with chronic periodontitis following phase 1 therapy. J. Evolution Med. Dent. Sci. 2019;8(52): 3977-3983, DOI: 


\section{BACKGROUND}

Periodontal disease is a microbial infection involving a variety of microbes that trigger inflammation, loss of connective tissue attachment and alveolar bone around the teeth. The primary etiologic factor of periodontitis is bacterial plaque. The bacteria involved are mainly of gram-negative species, express pathogenic factors that elicit host defence responses resulting in inflammation and tissue destruction. In fact, the propensity of periodontitis to proceed with periods of exacerbation and remission could suggest that the presence of other organisms contributes in the disease progression. ${ }^{1}$ The development of periodontitis may depend upon cooperative interactions among specific pathogenic bacteria and tissue destructive inflammatory mediators. Various evidences has shown relationship between type 2 diabetes and periodontal disease. ${ }^{2}$ However, the effect of periodontal intervention on these elements is yet to be ascertained.

Diabetes is a group of metabolic diseases characterized by hyperglycaemia resulting from defects in insulin secretion, insulin action, or both. The chronic hyperglycaemia of diabetes is associated with long-term damage, dysfunction, and failure of different organs, especially the eyes, kidneys, nerves, heart, and blood vessels. Several pathogenic processes are involved in the development of diabetes. These range from autoimmune destruction of the $\beta$-cells of the pancreas with consequent insulin deficiency to abnormalities that result in resistance to insulin action. Deficient insulin action results from inadequate insulin secretion and/or diminished tissue responses to insulin at one or more points in the complex pathways of hormone action. Impairment of insulin secretion and defects in insulin action frequently coexist in the same patient, and it is often unclear which abnormality, if either alone, is the primary cause of the hyperglycaemia.

At present, a bidirectional pathway has been suggested between DM and periodontitis, since patients with DM are more predisposed to periodontal diseases, the established periodontitis may simultaneously impair adequate glycaemic control. ${ }^{3}$ Moreover, poorly controlled diabetic subjects, presenting elevated levels of glycosylated haemoglobin (HbA1c), show higher attachment and alveolar bone loss and local inflammatory cytokines than well controlled patients 3,4 Exacerbated local inflammation in diabetic subjects with inadequate glycaemic control could modify the subgingival environment, and consequently, the subgingival microbial profile. Evidence has shown variations in the micro flora in non-diabetic and diabetic individuals; and also, within the diabetic individuals as well, based on their glycaemic control.5,6

We wanted to assess, compare and correlate the change in bacterial pathogen concentrations in plaque as confirmed polymerize chain reaction (PCR) in patients with and without type- 2 diabetes before and after phase- 1 therapy in patients with chronic periodontitis.

\section{METHODS}

This study was conducted among 120 patients of chronic periodontitis visiting the Department of Periodontics, Maharishi Markandeshwar College of Dental Sciences and Research, Mullana, Ambala and Department of Medicine, Maharishi Markandeshwar Medical College, Mullana Ambala. Ethical approval was obtained.

\section{Method of Collection of Data}

In this study, which was of 4 months duration, following initial screening, 120 patients with chronic periodontitis (Mild to moderate) were divided into 2 main groups based on their glycaemic status. Glycated haemoglobin levels were assessed using standardized assay- High Pressure Liquid Chromatography (HPLC).

\section{Group-1-Control $(n=30)$}

30 non-diabetic patients with chronic periodontitis.

\section{Group-2-Test: $(n=9$ 0)}

These 90 diabetic patients were further subdivided into 3 subgroups-

- 2 (A)- 30 diabetic patients with chronic periodontitis. (Good control $\left(\mathrm{HbA}_{1} \mathrm{C}<5 \%\right)$ )

- 2 (B)- 30 diabetic patients with chronic periodontitis. (Moderate control $\left(\mathrm{HbA}_{1} \mathrm{c}<7 \%\right)$ )

- 2 (C)- 30 diabetic patients with chronic periodontitis (Poor control $\left(\mathrm{HbA}_{1} \mathrm{c}>8 \%\right.$ ))

\section{Inclusion Criteria}

- Patients with chronic periodontitis (mild to moderate with up to $4 \mathrm{~mm}$ (CAL) clinical attachment loss and with the presence of true periodontal pocket (up to $6 \mathrm{~mm}$ ) in $\geq 30 \%$ of sites in the mouth.

- $\quad$ Systemically healthy patients (for Group 1).

- Diabetic patients having chronic periodontitis with varied levels of glycaemic control classified as good, moderate and poor (Group 2).

\section{Exclusion Criteria}

- $\quad$ Patients with any systemic diseases (Group 1)

- $\quad$ Presence of any systemic or debilitating diseases apart from diabetes in group.

The following parameters were assessed in all the patients at baseline (0), 1 months and 4 months

1) Clinical Parameters

1. Gingival index (Loe and Silness).(1963)(GI)

2. Plaque index (Silness and Loe).(1967)(PI)

3. Probing Pocket Depth (PPD) and relative Attachment Level (RAL)

2) Microbiological Parameters

1. Subgingival plaque samples were collected from the deepest part of the pocket by using a sterile curette in 2 different Eppendorf tubes containing tris-EDTA buffer.

2. The plaque samples were then sent for detecting bacteria (P. gingivalis, T. forsythia, P. intermedia, ) 


\section{Treatment Procedure}

Clinical Aspect- All the 120 patients were subjected to phase 1 therapy. After assessing the clinical parameters ultrasonic scaling was done followed by thorough root planing at baseline. Patients were recalled after 1 month and 4 months during which time, plaque samples were collected, and clinical parameters were recorded.

Microbiological Aspect- The plaque samples were subjected to PCR. The main components of the PCR Master Mix were HemoklenTaq buffer 5x, dNTP (2.5 mM), HaemoklenTaq enzyme and PCR water. Additives like DMSO, glycerol and formamide were also added for getting better results.

\section{PCR Protocol}

For PCR, bacterial DNA from the plaque samples was amplified in $50 \mu \mathrm{l}$ of a reaction mixture containing $3 \mu \mathrm{l}$ of the DNA sample, 1X PCR buffer ( $50 \mathrm{mMKCl}, 1.5 \mathrm{mMMgClz}, 10 \mathrm{mM}$ Tris- $\mathrm{HCl}, \mathrm{pH}$ 9.0), $2.5 \mathrm{mM}$ deoxyribonucleotide mixture $1 \mu \mathrm{l}$ of each primer, and 0.5-1 $\mu$ l of haemoklenTaq DNA polymerase (NAB) in a thermal cycler for 30 cycles. After amplification, $20 \mu \mathrm{l}$ of the PCR product was analyzed by agarose gel electrophoresis by incorporating propidium iodide on $1 \%$ gel. The newly synthesized DNA fragments were then visualized under ultraviolet light. The size of the PCR products was estimated from the electrophoretic migration of products relative to a $1 \mathrm{~kb}$ ladder marker. (Thermoscientific).

\section{Statistical Analysis}

All the clinical, microbiological and biochemical parameters recorded were statistically analysed using SPSS software version 13, and Paired t test.

\section{RESULTS}

\section{P. gingivalis (PG)- Intra Group (Table 1A)}

Group-1 (Control)- The mean difference in P. gingivalis counts between baseline and 1 month was $4926000000.0 \pm$ 5081385637.78 and baseline and 4 months was $5010000000.0 \pm 5069972454.52$ and 1 month and 4 months was $84000000.0+179031879.27$. This difference was not found to be statistically significant $(\mathrm{p}<0.05)$ at all-time intervals i.e. from baseline to 1 month, baseline to 4 months \& from 1 month to 4 months.

Group-2A (Test)- The mean difference in P. gingivalis counts between baseline and 1 month was 4286666666.67士 4992665885.44 and baseline and 4 months was $4312000000.0 \pm 5016730904.23$ and 1 month and 4 months was $25333333.33+43607444.27$. This difference was found to be statistically significant $(\mathrm{p}<0.05)$ at all-time intervals i.e. from baseline to 1 month, baseline to 4 months \& from 1 month to 4 months.

Group-2B (Test)- The mean difference in P. gingivalis counts between baseline and 1 month was $4677000000.00 \pm 4902599697.42$ and baseline and 4 months was $4763666666.67 \pm 4978032074.36$ and 1 month and 4 months was $86666666.67+238346796.50$. This difference was found to be statistically significant $(p<0.05)$ at all-time intervals i.e. from baseline to 1 month, baseline to 4 months \& from 1 month to 4 months.

\begin{tabular}{|c|c|c|c|c|c|}
\hline 泀 & 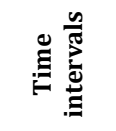 & $\underset{\Sigma}{\stackrel{\Xi}{\Xi}}$ & 芯 & हैं & : \\
\hline \multirow{3}{*}{ 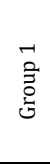 } & $\begin{array}{c}\text { Baseline - } \\
1 \text { month }\end{array}$ & 4926000000.0000000 & 5081385637.7960530 & .000 & S \\
\hline & $\begin{array}{l}\text { Baseline - } \\
4 \text { months }\end{array}$ & 5010000000.0000000 & 5069972454.5262740 & .000 & S \\
\hline & $\begin{array}{l}1 \text { month- } \\
4 \text { months } \\
\end{array}$ & 84000000.000000 & 179031879.264849 & .000 & S \\
\hline \multirow{3}{*}{$\begin{array}{l}\mathbb{\jmath} \\
\vdots \\
\vdots \\
0 \\
\dot{3}\end{array}$} & $\begin{array}{c}\text { Baseline - } \\
1 \text { month }\end{array}$ & 4286666666.6666665 & 4992665885.4441830 & .000 & S \\
\hline & $\begin{array}{l}\text { Baseline - } \\
4 \text { months }\end{array}$ & 4312000000.0000000 & 5016730904.2360690 & .000 & S \\
\hline & $\begin{array}{l}1 \text { month - } \\
4 \text { months }\end{array}$ & 25333333.333333 & 43607444.265885 & .000 & S \\
\hline \multirow{3}{*}{$\begin{array}{l}\text { Nै } \\
\stackrel{\infty}{0} \\
\text { Dें }\end{array}$} & $\begin{array}{c}\text { Baseline - } \\
1 \text { month }\end{array}$ & 4677000000.0000000 & 4902599697.4159990 & .000 & S \\
\hline & $\begin{array}{l}\text { Baseline - } \\
4 \text { months }\end{array}$ & 4763666666.6666670 & 4978032074.3576310 & .000 & S \\
\hline & $\begin{array}{l}1 \text { month - } \\
4 \text { months }\end{array}$ & 86666666.666667 & 238346796.501021 & .05 & S \\
\hline \multirow{3}{*}{ 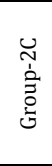 } & $\begin{array}{c}\text { Baseline - } \\
1 \text { month }\end{array}$ & 6844333333.3333330 & 4733278140.3613910 & .000 & S \\
\hline & $\begin{array}{l}\text { Baseline - } \\
4 \text { months }\end{array}$ & 6731000000.0000000 & 4727797001.0099430 & .000 & S \\
\hline & $\begin{array}{l}1 \text { month - } \\
4 \text { months }\end{array}$ & -113333333.333333 & 503363400.182888 & .227 & N.S \\
\hline \multicolumn{6}{|c|}{ Table 1A. Inter Group Comparison of P. gingivali } \\
\hline
\end{tabular}

Group-2C (Test): The mean difference in P. gingivalis counts between baseline and 1 month was 6844333333.33 4733278140.37 and baseline and 4 months was $6731000000.00 \pm 4727797001.00$ and 1 month and 4 months was $-113333333.33 \pm 503363400.19$. This difference was found to be statistically significant $(\mathrm{p}<0.05)$ from baseline to 1 month and baseline to 4 months but not from 1 month to 4 months, $(\mathrm{p}>0.05)$.

\section{P. gingivalis (PG)- Inter Group (Table 1B)}

Intergroup comparisons of Pg counts at the end of 1 month showed no significant differences between group 1 and $2 \mathrm{~A}$, $2 \mathrm{~B}$ and $2 \mathrm{C}$ and between groups $2 \mathrm{~A}$ and $2 \mathrm{~B}$ and $2 \mathrm{~A}$ and $2 \mathrm{C}$ and also between $2 \mathrm{~B}$ and $2 \mathrm{C},(\mathrm{p}>0.05)$. In contrast, at the end of 4 months, significant reduction was observed in group 1 when compared with group $2 \mathrm{C}$, group $2 \mathrm{~A}$ in comparison with group $2 \mathrm{C}$ and group $2 \mathrm{~B}$ when compared with group $2 \mathrm{C}(\mathrm{p}<0.05)$ However, no significant differences in $\mathrm{Pg}$ counts were observed between group 1 and $2 \mathrm{~A}$, group 1 and $2 \mathrm{~B}$ and also between group $2 \mathrm{~A}$ and $2 \mathrm{~B}(\mathrm{p}>0.05)$ at the end of 4 months.

\begin{tabular}{|c|c|c|c|c|c|c|}
\hline 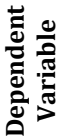 & 芯 & 瓷 & 芯 & 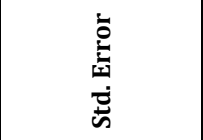 & $\ddot{b}$ & 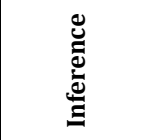 \\
\hline \multirow{6}{*}{ 心 } & \multirow{3}{*}{$\rightarrow$} & $2 \mathrm{~A}$ & 40666666.666667 & 59457412.557611 & 1.000 & $\mathrm{NS}[1 \mathrm{v} / \mathrm{s} 2 \mathrm{~A}]$ \\
\hline & & $2 \mathrm{~B}$ & -5666666.666667 & 59457412.557611 & 1.000 & $\mathrm{NS}[1 \mathrm{v} / \mathrm{s} 2 \mathrm{~B}]$ \\
\hline & & $2 \mathrm{C}$ & -68333333.333333 & \begin{tabular}{|l|}
59457412.557611 \\
\end{tabular} & 1.000 & $\mathrm{NS}[1 \mathrm{v} / \mathrm{s} \mathrm{2C}]$ \\
\hline & \multirow{2}{*}{$\mathbb{s}$} & $2 \mathrm{~B}$ & -46333333.333333 & 59457412.557611 & 1.000 & $\mathrm{NS}[2 \mathrm{Av} / \mathrm{s} 2 \mathrm{~B}]$ \\
\hline & & $2 \mathrm{C}$ & 109000000.000000 & 59457412.557611 & .416 & $\mathrm{NS}[2 \mathrm{~A} v / \mathrm{s} 2 \mathrm{C}]$ \\
\hline & $2 \mathrm{~B}$ & $2 \mathrm{C}$ & -62666666.666667 & 59457412.557611 & 1.000 & $\mathrm{NS}[2 \mathrm{~B} v / \mathrm{s} 2 \mathrm{C}]$ \\
\hline \multirow{6}{*}{ ৩ } & \multirow{3}{*}{1} & $2 \mathrm{~A}$ & -18000000.0000 & 58166329.0838 & 1.000 & $\mathrm{NS}[1 \mathrm{v} / \mathrm{s} 2 \mathrm{~A}]$ \\
\hline & & 2B & -3000000.0000 & 58166329.0838 & 1.000 & $\mathrm{NS}[1 \mathrm{v} / \mathrm{s} 2 \mathrm{~B}]$ \\
\hline & & $2 \mathrm{C}$ & $-265666666.6667^{*}$ & 58166329.0838 & .000 & $\mathrm{~S}[1 \mathrm{v} / \mathrm{s} 2 \mathrm{C}]$ \\
\hline & \multirow{2}{*}{$2 \mathrm{~A}$} & 2B & 15000000.0000 & 58166329.0838 & 1.000 & $\mathrm{NS}[2 \mathrm{Av} / \mathrm{s} 2 \mathrm{~B}]$ \\
\hline & & $2 \mathrm{C}$ & $-247666666.6667^{*}$ & 58166329.0838 & .000 & $\mathrm{~S}[2 \mathrm{~A} v / \mathrm{s} 2 \mathrm{C}]$ \\
\hline & 2B & $2 \mathrm{C}$ & $-262666666.6667^{*}$ & 58166329.0838 & .000 & $\mathrm{~S}[2 \mathrm{~B} \mathrm{v} / \mathrm{s} 2 \mathrm{C}]$ \\
\hline \multicolumn{7}{|c|}{ Table 1b. Inter Group Comparison of P. gingivalis } \\
\hline
\end{tabular}




\section{T. forsythia (TF)- Intra Group (Table 2A)}

Group-1 (Control): The mean difference in T. forsythia scores

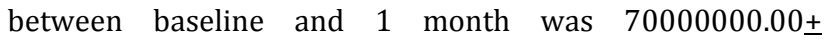
255653321.30, at baseline and 4 months was $76333333.33 \pm 252675112.83$ and 1 month and 4 months was $6333333.33 \pm 24280449.54$. This difference was not found to be statistically significant $(p>0.05)$ at all time intervals i.e. from baseline to 1 month, baseline to 4 months and 1 month and 4 months.

Group-2A (Test): The mean difference in T. forsythia scores between baseline and 1 month was 1995333333.33+ 4042906715.80, at baseline and 4 months was $1696551724.13 \pm 3768050154.98$ and 1 month and 4 months was $-23103448.28 \pm 170589386.89$. This difference was found to be statistically significant $(p<0.05)$ from baseline to 1 month and baseline to 4 months but not from 1 month to 4 months, $(\mathrm{p}>0.05)$.

Group-2B (Test): The mean difference in T. forsythia scores between baseline and 1 month was $1626000000.00 \pm 3617409627.60$, at baseline and 4 months was $1663333333.33 \pm 3699998446.72$ and 1 month and 4 months was $37333333.33 \pm 182736541.71$. This difference was found to be statistically significant $(p<0.05)$ from baseline to 1 month and baseline to 4 months but not from 1 month to 4 months, $(\mathrm{p}>0.05)$.

Group-2C (Test): The mean difference in T. forsythia scores between baseline and 1 month was $1099633333.33 \pm 3020280170.64$, at baseline and 4 months was $1044000000.00 \pm 3052032222.90$ and 1 month and 4 months was $-55633333.33 \pm 243356483.70$. This difference was found to be statistically significant $(\mathrm{p}<0.05)$ at baseline to 1 month but not from baseline to 4 months \& from 1 month to 4 months, $(p>0.05)$.

\begin{tabular}{|c|c|c|c|c|c|}
\hline 芯 & 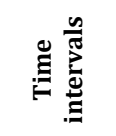 & 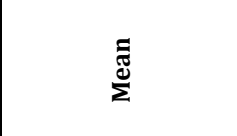 & 总 & in & 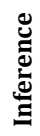 \\
\hline \multirow{3}{*}{$\begin{array}{l}\overrightarrow{0} \\
\text { डे }\end{array}$} & $\begin{array}{c}\text { Baseline - } \\
1 \text { month }\end{array}$ & 70000000.0000000 & 255653321.2959597 & .144 & NS \\
\hline & $\begin{array}{l}\text { Baseline - } \\
4 \text { months }\end{array}$ & 76333333.3333333 & 252675112.8300493 & .109 & NS \\
\hline & $\begin{array}{l}1 \text { month - } \\
4 \text { months }\end{array}$ & 6333333.333333 & 24280449.540424 & .164 & NS \\
\hline \multirow{3}{*}{ 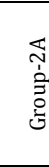 } & $\begin{array}{c}\text { Baseline - } \\
1 \text { month }\end{array}$ & 1995333333.3333335 & 4042906715.7978890 & .011 & $\mathrm{~S}$ \\
\hline & $\begin{array}{l}\text { Baseline - } \\
4 \text { months }\end{array}$ & 1696551724.1379310 & 3768050154.9798074 & .022 & $S$ \\
\hline & $\begin{array}{l}1 \text { month - } \\
4 \text { months }\end{array}$ & -23103448.275862 & 170589386.880474 & .472 & NS \\
\hline \multirow{3}{*}{ 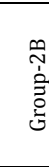 } & $\begin{array}{l}\text { Baseline - } \\
1 \text { month }\end{array}$ & 1626000000.0000000 & 3617409627.5916977 & .020 & S \\
\hline & $\begin{array}{l}\text { Baseline - } \\
4 \text { months }\end{array}$ & 1663333333.3333333 & 3699998446.7222586 & .020 & S \\
\hline & $\begin{array}{l}1 \text { month - } \\
4 \text { months }\end{array}$ & 37333333.333333 & 182736541.715555 & .272 & NS \\
\hline \multirow{3}{*}{ 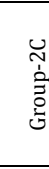 } & $\begin{array}{c}\text { Baseline - } \\
1 \text { month }\end{array}$ & 1099633333.3333333 & 3020280170.6456640 & .056 & $\mathrm{~S}$ \\
\hline & $\begin{array}{l}\text { Baseline - } \\
4 \text { months }\end{array}$ & 1044000000.0000000 & 3052032222.9057760 & .071 & NS \\
\hline & $\begin{array}{l}1 \text { month - } \\
4 \text { months }\end{array}$ & -55633333.333333 & 243356483.704297 & .221 & N.S \\
\hline \multicolumn{6}{|c|}{ Table 2a. Intra Group Comparison of T. forsyth } \\
\hline
\end{tabular}

\section{T. forsythia (TF)- Inter Group (Table 2B)}

Inter group comparisons of Tf counts at the end of 1 month showed no significant differences between group 1 and $2 \mathrm{~A}$, $2 \mathrm{~b}$ and $2 \mathrm{C}$ respectively and also between group $2 \mathrm{~A}$ and $2 \mathrm{~B}$ and $2 \mathrm{~A}$ and $2 \mathrm{C}$ and also between groups $2 \mathrm{~B}$ and $2 \mathrm{C}$. ( $\mathrm{p}>0.05)$. Similarly, no significant differences were found between group 1 and $2 \mathrm{~A}$ and $2 \mathrm{~B}$ respectively, and group $2 \mathrm{~A}$ and $2 \mathrm{~B}$ and $2 \mathrm{~A}$ and $2 \mathrm{C}$ and also group $2 \mathrm{~B}$ and $2 \mathrm{C}$ at the end of 4 months, $(p>0.05)$.In contrast, significant difference was found in the T.F counts at the end of 4 months between group 1 and group $2 \mathrm{C}(\mathrm{p}<0.05)$

\begin{tabular}{|c|c|c|c|c|c|c|}
\hline 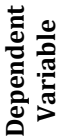 & 苍 & 芌 & 芯 & 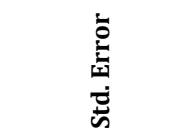 & D' & 总 \\
\hline \multirow{6}{*}{ 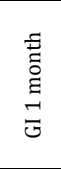 } & \multirow{3}{*}{1} & $2 \mathrm{~A}$ & 6000000.000000 & 33543266.792703 & 1.000 & $\mathrm{NS}[1 \mathrm{v} / \mathrm{s} 2 \mathrm{~A}]$ \\
\hline & & $2 \mathrm{~B}$ & -56666666.666667 & 33543266.792703 & .563 & $\mathrm{NS}[1 \mathrm{v} / \mathrm{s} 2 \mathrm{~B}]$ \\
\hline & & $2 \mathrm{C}$ & 2966666.666667 & 33543266.792703 & 1.000 & $\mathrm{NS}[1 \mathrm{v} / \mathrm{s} 2 \mathrm{C}]$ \\
\hline & \multirow[b]{2}{*}{$2 \mathrm{~A}$} & $2 \mathrm{~B}$ & -62666666.666667 & 33543266.792703 & .386 & $\mathrm{NS}[2 \mathrm{Av} / \mathrm{s} 2 \mathrm{~B}]$ \\
\hline & & $2 \mathrm{C}$ & -3033333.333333 & 33543266.792703 & 1.000 & $\mathrm{NS}[2 \mathrm{~A} v / \mathrm{s} 2 \mathrm{C}]$ \\
\hline & 2B & $2 \mathrm{C}$ & \begin{tabular}{|l|}
59633333.333333 \\
\end{tabular} & 33543266.792703 & .468 & $\mathrm{NS}[2 \mathrm{~B} \mathrm{v} / \mathrm{s} 2 \mathrm{C}]$ \\
\hline \multirow{6}{*}{ 芑 } & \multirow{3}{*}{1} & $2 \mathrm{~A}$ & -23482758.620690 & 47492484.013034 & 1.000 & $\mathrm{NS}[1 \mathrm{v} / \mathrm{s} 2 \mathrm{~A}]$ \\
\hline & & $2 \mathrm{~B}$ & -25666666.666667 & 47088285.309193 & 1.000 & $\mathrm{NS}[1 \mathrm{v} / \mathrm{s} 2 \mathrm{~B}]$ \\
\hline & & $2 \mathrm{C}$ & -59000000.000000 & 47088285.309193 & 1.000 & $\mathrm{~S}[1 \mathrm{v} / \mathrm{s} 2 \mathrm{C}]$ \\
\hline & \multirow{2}{*}{$2 \mathrm{~A}$} & $2 \mathrm{~B}$ & \begin{tabular}{|l|}
-2183908.045977 \\
\end{tabular} & 47492484.013034 & 1.000 & $\mathrm{NS}[2 \mathrm{Av} / \mathrm{s} 2 \mathrm{~B}]$ \\
\hline & & $2 \mathrm{C}$ & -35517241.379310 & 47492484.013034 & 1.000 & $\mathrm{NS}[2 \mathrm{~A} v / \mathrm{s} 2 \mathrm{C}]$ \\
\hline & 2B & $2 \mathrm{C}$ & -33333333.333333 & 47088285.309193 & 1.000 & $\mathrm{NS}[2 \mathrm{~B} v / \mathrm{s} 2 \mathrm{C}]$ \\
\hline \multicolumn{7}{|c|}{ Table $2 b$. Inter Group Comparison of T. forsythia } \\
\hline
\end{tabular}

\section{P. intermedia (PI)- Intra Group (Table 3A, Graph 3A)}

Group-1 (control): The mean difference in P. intermedia counts between baseline and 1 month was $1755333333.33 \pm$ 3731564092.27, at baseline and 4 months was $1775000000.00+3750355155.60$ and 1 month and 4 months was $19666666.67+37644739.82$. This difference was found to be statistically significant $(\mathrm{p}<0.05)$ at all time intervals i.e. from baseline to 1 month, baseline to 4 months \& from 1 month to 4 months.

Group-2A (Test): The mean difference in P. intermedia counts between baseline and 1 month was $1821333333.33 \pm$ 3716936756.33, at baseline and 4 months was $1799666666.67 \pm 3652283634.53$ and 1 month and 4 months was $-21666666.67 \pm 310428883.90$. This difference was found to be statistically significant $(p<0.05)$ at baseline to 1 month and baseline to 4 months but not from 1 month to 4 months, $(\mathrm{p}>0.05)$.

Group-2B (Test): The mean difference in P. intermedia counts between baseline and 1 month was $2124666666.67 \pm 3927763014.60$, at baseline and 4 months was $2169000000.00 \pm 3988301297.71$ and 1 month and 4 months was $44333333.33+184216576.69$. This difference was found to be statistically significant $(p<0.05)$ at baseline to 1 month, baseline to 4 months but not from 1 month to 4 months, $(p>0.05)$.

Group-2C (Test): The mean difference in $P$. intermedia counts between baseline and 1 month was $287266670.30 \pm 421938784.74$, at baseline and 4 months was $292633336.97 \pm 501342091.10$ and 1 month and 4 months was $5366666.67 \pm 370736404.49$. This difference was found to be statistically significant $(p<0.05)$ at baseline 
to 1 month, baseline to 4 months but not from 1 month to 4 months, $(\mathrm{p}>0.05)$.

\section{P. intermedia (PI)- Inter Group (Table 3B, Graph 3B)}

Inter group comparisons of Pi counts revealed no significant differences between all the four groups at the end of 1 month and 4 months respectively, $(p>0.05)$.

\begin{tabular}{|c|c|c|c|c|c|}
\hline 气̊. & 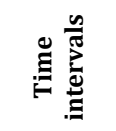 & 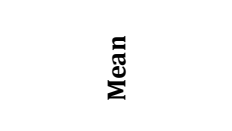 & 总 & $\ddot{n}$ & 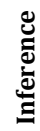 \\
\hline \multirow{3}{*}{$\begin{array}{l}\overrightarrow{0} \\
\hat{0} \\
\dot{3}\end{array}$} & $\begin{array}{c}\text { Baseline - } \\
1 \text { month }\end{array}$ & 1755333333.3333333 & 3731564092.2691710 & .015 & S \\
\hline & $\begin{array}{l}\text { Baseline - } \\
4 \text { months }\end{array}$ & 1775000000.0000000 & 3750355155.5957274 & .015 & S \\
\hline & $\begin{array}{l}1 \text { month- } \\
4 \text { months }\end{array}$ & 19666666.666667 & 37644739.828847 & .008 & S \\
\hline \multirow{3}{*}{ 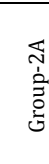 } & $\begin{array}{c}\text { Baseline - } \\
1 \text { month }\end{array}$ & 1821333333.3333333 & 3716936756.3323855 & 012 & $S$ \\
\hline & $\begin{array}{l}\text { Baseline - } \\
4 \text { months }\end{array}$ & 17996666666.6666667 & 3652283634.5396880 & 011 & $\mathrm{~S}$ \\
\hline & $\begin{array}{l}1 \text { month- } \\
4 \text { months }\end{array}$ & -21666666.666667 & 310428883.891340 & .705 & NS \\
\hline \multirow{3}{*}{ 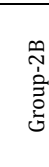 } & $\begin{array}{c}\text { Baseline - } \\
1 \text { month }\end{array}$ & 2124666666.6666665 & 3927763014.5988407 & .006 & S \\
\hline & $\begin{array}{l}\text { Baseline - } \\
4 \text { months }\end{array}$ & 2169000000.0000000 & 3988301297.7180290 & .006 & S \\
\hline & $\begin{array}{l}1 \text { month- } \\
4 \text { months }\end{array}$ & 44333333.333333 & 184216576.687433 & .198 & NS \\
\hline \multirow{3}{*}{ 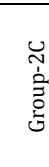 } & $\begin{array}{c}\text { Baseline - } \\
1 \text { month }\end{array}$ & 287266670.3000000 & 421938784.7430390 & .001 & S \\
\hline & $\begin{array}{l}\text { Baseline - } \\
4 \text { months }\end{array}$ & 292633336.9666666 & 501342091.9915900 & .003 & S \\
\hline & $\begin{array}{l}1 \text { month- } \\
4 \text { months }\end{array}$ & 5366666.6666667 & 370736404.483287 & .937 & N.S \\
\hline
\end{tabular}

\begin{tabular}{|c|c|c|c|c|c|c|}
\hline 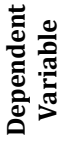 & 苛 & 气̊. & 芯 & 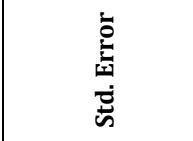 & in & 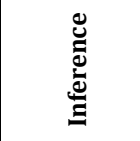 \\
\hline \multirow{6}{*}{ ৩ } & \multirow{3}{*}{1} & $2 \mathrm{~A}$ & -24000000.000000 & 46950335.706878 & 1.000 & $\mathrm{NS}[1 \mathrm{v} / \mathrm{s} 2 \mathrm{~A}]$ \\
\hline & & $2 \mathrm{~B}$ & -28333333.333333 & \begin{tabular}{|l|}
46950335.706878 \\
\end{tabular} & 1.000 & $\mathrm{NS}[1 \mathrm{v} / \mathrm{s} 2 \mathrm{~B}]$ \\
\hline & & $2 \mathrm{C}$ & -51400000.000000 & 46950335.706878 & 1.000 & $\mathrm{NS}[1 \mathrm{v} / \mathrm{s} 2 \mathrm{C}]$ \\
\hline & \multirow[b]{2}{*}{$2 \mathrm{~A}$} & $2 \mathrm{~B}$ & -4333333.333333 & 46950335.706878 & 1.000 & $\mathrm{NS}[2 \mathrm{Av} / \mathrm{s} 2 \mathrm{~B}]$ \\
\hline & & $2 \mathrm{C}$ & -27400000.000000 & 46950335.706878 & 1.000 & $\mathrm{NS}[2 \mathrm{~A} v / \mathrm{s} 2 \mathrm{C}]$ \\
\hline & 2B & $2 \mathrm{C}$ & -23066666.666667 & \begin{tabular}{|l|l|}
46950335.706878 \\
\end{tabular} & 1.000 & $\mathrm{NS}[2 \mathrm{~B} \mathrm{v} / \mathrm{s} 2 \mathrm{C}]$ \\
\hline \multirow{6}{*}{ 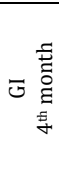 } & \multirow{3}{*}{1} & $2 \mathrm{~A}$ & -65333333.333333 & 446424079.867789 & .972 & $\mathrm{NS}[1 \mathrm{v} / \mathrm{s} 2 \mathrm{~A}]$ \\
\hline & & $2 \mathrm{~B}$ & -3666666.666667 & 46424079.867789 & 1.000 & $\mathrm{NS}[1 \mathrm{v} / \mathrm{s} 2 \mathrm{~B}]$ \\
\hline & & $2 \mathrm{C}$ & -65700000.000000 & 46424079.867789 & .958 & $\mathrm{NS}[1 \mathrm{v} / \mathrm{s} 2 \mathrm{C}]$ \\
\hline & \multirow{2}{*}{$2 \mathrm{~A}$} & 2B & 61666666.666667 & 46424079.867789 & 1.000 & $\mathrm{NS}[2 \mathrm{Av} / \mathrm{s} 2 \mathrm{~B}]$ \\
\hline & & $2 \mathrm{C}$ & -366666.666667 & 46424079.867789 & 1.000 & $\mathrm{NS}[2 \mathrm{~A} v / \mathrm{s} 2 \mathrm{C}]$ \\
\hline & $2 \mathrm{~B}$ & $2 \mathrm{C}$ & -62033333.333333 & 46424079.867789 & 1.000 & $\mathrm{NS}[2 \mathrm{~B} \mathrm{v} / \mathrm{s} 2 \mathrm{C}]$ \\
\hline
\end{tabular}

\section{DISCUSSION}

Periodontitis usually results from the complex interactions of the microbial communities with the host elements in the gingival sulcus that leads to inflammation, loss of connective tissue attachment and alveolar bone around the teeth. ${ }^{6}$

Diabetes mellitus (DM) is well recognized as a risk factor for periodontal disease, while periodontitis is thought to influence the systemic inflammatory condition, lipid and glucose metabolism and insulin resistance. ${ }^{7}$ Diabetes mellitus, especially when uncontrolled, appears to be an important risk factor for periodontal destruction, since an alteration in host-response and microbiological aspects occurs in diabetic subjects. ${ }^{8}$ Periodontal disease leads to an increase in insulin resistance, which could impair glycaemic control. Thus, the control of periodontal disease is necessary for better systemic health in these individuals. Periodontal treatment relies on biofilm disruption and plaque control to prevent recolonization and recurrence of the disease. ${ }^{8}$

The relationship between plaque accumulation and $\mathrm{HbA}_{1} \mathrm{c}$ levels remains controversial. ${ }^{9-11}$ Some reports have observed an improved compliance with oral hygiene amongst diabetic subjects with better glycaemic control, while others have not found this association.12-14 Some studies have shown the impact of type 1 and type $2 \mathrm{DM}$ on tooth loss, ${ }^{15}$ but the association between metabolic control and the number of remaining teeth has not been considered. Previous studies have shown that the microbiota associated with diabetes does not appear different from the microbiota of non-diabetic patients.15-19 Although the microbiology of periodontal disease in DM is fairly well understood. The sample size of 120 patients evaluated in this study was arrived at following consultation with the statistician which was in accordance with the vast majority of clinical periodontal treatment studies in humans. ${ }^{20}$ In order to understand this phenomenon with better sensitivity and specificity, it was decided to carry out the study by dividing the 120 patients into 4 groups based on the level of glycaemic control.

The clinical parameters (GI, PI, PPD, RAL) were assessed to evaluate the disease severity at regular intervals and also to understand whether phase- 1 therapy has an effect on patients with chronic periodontitis with and without diabetes.

The gold standard for recording changes in periodontal status is the longitudinal measurements with relative attachment level (RAL) from CEJ to the base of the pocket. Due to the relative inconsistencies in determining CEJ accurately at the selected sites, it was decided to use a customized acrylic stent and use the base of the stent as the fixed reference point and evaluate relative attachment level (RAL).

In addition, the microbiological analysis with the help of multiplex PCR technique was done to evaluate the effect of phase- 1 therapy on bacterial concentrations in patients with chronic periodontitis with and without diabetes. Single-stage PCR was used to detect $P$. gingivalis, $P$. intermedia, and $T$. forsythia. It has been suggested that pooling of plaque samples increases the probability of detecting existing pathogens. ${ }^{21}$

Intergroup comparison revealed, a significant improvement in glycated $\mathrm{Hb}$ levels in the non-diabetic group as compared to the diabetic groups at 1 month following phase-1 therapy. Additionally significant improvement was also observed in the well-controlled and moderately controlled diabetic group when compared with the poorly controlled group. This is in accordance with Taylor et al 2008,22 Shlossman $1990^{23}$ who concluded that phase-1 therapy improves the glycaemic status of diabetic patients with chronic periodontitis.

With regard to the microbiologic parameters, intra group comparison revealed a significant improvement in Pg counts, Tf count, Pi count, count with in both the test (Diabetic) and control (non-diabetic) group from baseline to 1 month and 4 months except in poorly controlled diabetic group at 1 month and 4 months. This is in accordance with the findings of Sandholm et al 198924, Contreras at al 199925, Saygun et al 
$2008^{26}$ who concluded that phase-1 therapy results in reduction of bacterial counts in patients with chronic periodontitis and diabetes and also with Hintao et al 200727, IbrahimuMdala $2013^{28}$ who concluded that phase- 1 therapy results in reduction of bacterial counts in patients with chronic periodontitis

Inter group comparison revealed significant reduction in Pg counts, Tf count, Pi count, in the non-diabetic group compared to the poorly controlled diabetic group and well controlled diabetic group compared to poorly-controlled diabetic group at the end of 4 months. But interestingly no such differences were observed at 1 month. This is accordance with Makiura et al $2008^{29}$ who concluded that Pg counts were reduced in the patients with diabetes following phase-1 therapy.

\section{CONCLUSIONS}

In-vitro microbiological assessment (PCR analysis) showed significant reduction of bacteria within both diabetic and non-diabetic groups. In contrast, the intergroup comparison revealed no significant difference in the microbial counts in both the diabetic and non-diabetic groups thus suggesting that phase-1 therapy may not alter the pathogenic microflora in chronic periodontitis patients with or without diabetes. Correlation assessments of clinical parameters with the microbiological and biochemical parameters showed significant correlation at various time intervals again suggesting that phase 1 therapy results in improved clinical and microbiologic status and glycaemic status in chronic periodontitis patients with and without diabetes.

\section{REFERENCES}

[1] Dawson DR, Wang C, Danaher RJ, et al. Real time polymerase chain reaction to determine the prevalence and copy number of Epstein-Barr virus and cytomegalovirus DNA in subgingival plaque at individual healthy and periodontal disease sites. J Periodontol 2009;80(7):1133-1140.

[2] Kaur G, Holtfreter B, Rathmann W, et al. Association between type 1 and type 2 diabetes with periodontal disease and tooth loss. J Clin Periodontol 2009;36(9):765-74.

[3] Taylor GW. Bidirectional interrelationships between diabetes and periodontal diseases: an epidemiologic perspective. Ann Periodontol 2001;6(1):99-112.

[4] Safkan-Seppala B, Sorsa T, Tervahartiala T, et al. Collagenases in gingival crevicular fluid in type 1 diabetes mellitus. J Periodontol 2006;77(2):189-94.

[5] Chalabi M, Rezaie F, Moghim S, et al. Periodontopathic bacteria and herpesviruses in chronic periodontitis. Mol Oral Microbiol 2010;25(3):236-40.

[6] Socransky SS, Haffajee AD. Periodontal microbial ecology. Periodontol 2000 2005;38:135-87.

[7] Iacopino AM, Cutler CW. Pathophysiological relationships between periodontitis and systemic disease: recent concepts involving serum lipids. J Periodontol 2000;71(8):1375-84.
[8] Meenawat A, Punn K, Srivastava V, et al. Periodontal disease and type I diabetes mellitus: associations with glycaemic control and complications J Indian Soc Periodontol 2013;17(5):597-600.

[9] Teeuw WJ, Gerdes VE, Loos BG. Effect of periodontal treatment on glycaemic control of diabetic patients: a systematic review and meta-analysis. Diabetes Care 2010;33(2):421-7.

[10] Syrjala AM, Kneckt MC, Knuuttila ML. Dental self-efficacy as a determinant to oral health behaviour, oral hygiene and HbA1c level among diabetic patients. J Clin Periodontol 1999;26(9):616-21.

[11] Goncalves D, Correa FO, Khalil NM, et al. The effect of non-surgical periodontal therapy on peroxidase activity in diabetic patients: a case-control pilot study. J Clin Periodontol 2008;35(9):799-806.

[12] Chen L, Wei B, Li J, et al. Association of periodontal parameters with metabolic level and systemic inflammatory markers in patients with type 2 diabetes. J Periodontol 2010;81(3):364-71.

[13] Tervonen T, Karjalainen K. Periodontal disease related to diabetic status. A pilot study of the response to periodontal therapy in type 1 diabetes. J Clin Periodontol 1997;24(7):505-10.

[14] Kardesler L, Buduneli N, Cetinkalp S, et al. Adipokines and inflammatory mediators after initial periodontal treatment in patients with type 2 diabetes and chronic periodontitis. J Periodontol 2010;81(1):24-33.

[15] Katz PP, Wirthlin MR, Szpunar SM, et al. Epidemiology and prevention of periodontal disease in individuals with diabetes. Diabetes Care 1991;14(5):375-85.

[16] Yuan K, Chang CJ, Hsu PC, et al. Detection of putative periodontal pathogens in non-insulin-dependent diabetes mellitus and non-diabetes mellitus by polymerase chain reaction. J Periodontal Res 2001;36(1):18-24.

[17] Tervonen T, Oliver RC, Wol LF, et al. Prevalence of periodontal pathogens with varying metabolic control of diabetes mellitus. J Clin Periodontol 1994;21(6):375-9.

[18] Sbordone L, Ramaglia L, Barone A, et al. Periodontal status and selected cultivable anaerobic microflora of insulin dependent juvenile diabetics. J Periodontol 1995;66(6):452-61.

[19] Zambon JJ, Reynolds H, Fisher JG, et al. Microbiological and immunological studies of adult periodontitis in patients with noninsulin-dependent diabetes mellitus. J Periodontol 1988;59(1):23-31.

[20] Kaur G, Holtfreter B, Rathmann W, et al. Association between type 1 and type 2 diabetes with periodontal disease and tooth loss. J Clin Periodontol 2009;36(9):765-74.

[21] Kapp JM, Boren SA, Yun S, et al. Diabetes and tooth loss in a national sample of dentate adults reporting annual dental visits. Prev Chronic Dis 2007;4(3):A59.

[22] Taylor GW, Borgnakke WS. Periodontal disease: associations with diabetes, glycaemic control and complications. Oral Dis 2008;14(3):191-203.

[23] Shlossman M, Knowler WC, Pettitt DJ, et al. Type 2 diabetes mellitus and periodontal disease. J Am Dent Assoc 1990;121(4):532-36. 
[24] Sandholm L, Swanljung O, Rytomaa I, et al. Morphotypes of the subgingival microflora in diabetic adolescents in Finland. J Periodontol 1989;60(9):526-8.

[25] Contreras A, Umeda M, Chen C, et al. Relationship between herpesviruses and adult periodontitis and periodontopathic bacteria. J Periodontol 1999;70(5):478-84.

[26] Saygun I, Kubar A, Sachin S, et al. Quantitative analysis of association between herpes viruses and bacterial pathogens in periodontitis. J Periodontal Res 2008;43(3):352-9.

[27] Hintao J, Teanpaisan R, Chongsuvivatwong V, et al. The microbiological profiles of saliva, supragingival and subgingival plaque and dental caries in adults with and without type 2 diabetes mellitus. Oral Microbiol Immunol 2007;22(3):175-81.

[28] Mdala I, Olsen I, Haffajee AD, et al. Multilevel analysis of bacterial counts from chronic periodontitis after root planing/scaling, surgery, and systemic and local antibiotics: 2-year results. J Oral Microbiol 2013;5(1):20939.

[29] Makiura N, Ojima M, Kou Y, et al. Relationship of Porphyromonas gingivalis with glycaemic level in patients with type 2 diabetes following periodontal treatment. Oral Microbiol Immunol 2008;23(4):348-51. 